INPLASY

PROTOCOL

To cite: Zhang et al. Impact of cancer on mortality and severity of COVID-19: a protocol for systematic review and meta-analysis. Inplasy protocol 202090093. doi: 10.37766/inplasy2020.9.0093

Received: 25 September 2020

Published: 26 September 2020

Corresponding author:

Hao Han

2860721065@qq.com

Author Affiliation:

Department of Geriatric

Respiratory Medicine, First

Hospital of Lanzhou

University, Lanzhou, China

Support: Cuiying Scientific and Technol.

Review Stage at time of this

submission: Preliminary

searches.

Conflicts of interest:

None.

\section{Impact of cancer on mortality and severity of COVID-19: a protocol for systematic review and meta-analysis}

Zhang, Y1; Han, H²; Tian, Y3; Dong, J4; Yu, Y5.

Review question / Objective: To examine whether cancer patients with COVID-19 may be at an increased risk of severe illness and whether cancer is associated with mortality in patients with COVID-19.

Condition being studied: Cancer patients are considered a highly vulnerable population in the COVID-19 epidemic, but the relationship between cancer and the severity and mortality of patients with COVID-19 remains unclear. This study aimed to explore whether cancer patients with COVID-19 may be at an increased risk of severe illness and mortality.

INPLASY registration number: This protocol was registered with the International Platform of Registered Systematic Review and Meta-Analysis Protocols (INPLASY) on 26 September 2020 and was last updated on 26 September 2020 (registration number INPLASY202090093).

\section{INTRODUCTION}

Review question / Objective: To examine whether cancer patients with COVID-19 may be at an increased risk of severe illness and whether cancer is associated with mortality in patients with COVID-19.
Condition being studied: Cancer patients are considered a highly vulnerable population in the COVID-19 epidemic, but the relationship between cancer and the severity and mortality of patients with COVID-19 remains unclear. This study aimed to explore whether cancer patients 
with COVID-19 may be at an increased risk of severe illness and mortality.

\section{METHODS}

Participant or population: Patients have a laboratory-confirmed diagnosis of CovID-19.

Intervention: Patients with severe illness or non-survivors.

Comparator: Patients with non-severe illness or survivors.

Study designs to be included: Clinical studies with $\geq 10$ nonoverlapping patients diagnosed with COVID-19.

Eligibility criteria: We will include studies that meet the following criteria: (1) patients have a laboratory-confirmed diagnosis of COVID-19; (2) provided the prevalence of cancer between COVID-19 patients with severe or non-severe illness or between non-survivors and survivors; (3) with a sample size of larger than 10 patients; (4) published in Chinese and English. The following studies will be excluded: (1) studies did not report data related to cancer patients; (2) studies focused on only suspected cases or suspected cases and confirmed cases; (3) review articles, protocols, guidelines, consensus, comments, abstracts, letters, and editorials.

Information sources: We will perform comprehensive searches in PubMed, EMBASE.com, Web of Science, and the Cochrane Central Register of Controlled Trials (CENTRAL) up to September 30, 2020. We will use search terms include the following words: "severe acute respiratory syndrome coronavirus 2", "SARS-CoV-2", "coronavirus disease-19", "COVID-19", "novel corona virus", "new coronavirus", "2019-nCoV", "novel coronavirus", "nCoV-2019", "novel coronavirus pneumonia", "2019 novel coronavirus", "coronavirus disease 2019", "neoplasms", "neoplasia", "tumor", "tumour", "cancer", "malignancy", "clinical characteristic", "clinical feature", "risk factor", "prognosis", and "comorbidities". Reference lists of eligible studies and relevant systematic reviews will be also manually searched for potentially eligible studies.

Main outcome(s): Differences of cancer prevalence in COVID-19 patients with or without severe illness and non-survivors or survivors.

Additional outcome(s): None.

Data management: We will develop a standardized data extraction form using Microsoft Excel 2016 (Microsoft Corp, Redmond, WA, http://www.microsoft.com) through discussions with the review team and will revise it after piloting on a random of six studies. The data will be extracted include: (1) study characteristics (first author, year of publication, country of the corresponding author, journal name, publication language, study setting, recruitment time frame), population characteristics (age, sex, sample size), and outcomes of interest (number of cancer patients, sever cases, non-severe cases, deaths, and survivors). The severe illness is defined in this study as patients experiencing acute respiratory distress syndrome (ARDS), requiring mechanical ventilation, requiring vital life support, or requiring intensive care unit admission (ICU) support. The data extraction will be performed by one reviewer and checked by a second reviewer. Discrepancies will be resolved by consensus or by the discussion with a third reviewer.

Quality assessment / Risk of bias analysis: We will use the Newcastle-Ottawa quality assessment scale (NOS) to assess the quality of included studies. Studies that obtained more than 7 stars will be considered as high quality, 5-7 stars will be considered as moderate quality, and lower than 5 stars will be considered as low quality. Two reviewers will conduct quality assessment independently. Any conflicts will be resolved by consensus or by the discussion with a third reviewer.

Strategy of data synthesis: We will conduct pairwise meta-analyses to compute the 
odds ratio (OR) and $95 \%$ confidence interval $(\mathrm{Cl})$ of cancer prevalence in COVID-19 patients with or without severe illness, and non-survivors or survivors. The meta-analyses will be performed using the inverse variance method with the randomeffects model. The statistical heterogeneity will be assessed with the 12 statistic, and value of $<25 \%, 26-50 \%$, and $>50 \%$ will be considered as low, moderate, and high level of heterogeneity, respectively. All analyses will be conducted using Stata (13.0; Stata Corporation, College Station, Texas, USA Stata) and the statistical level of significance will be set at $P<0.05$.

Subgroup analysis: We plan to conduct subgroup analyses of the outcomes between different countries.

Sensibility analysis: Sensitivity analyses will be conducted by excluding studies with high risk of bias to assess the stability of results.

Country(ies) involved: China.

Keywords: COVID-19; Neoplasms; Prevalence; Severe illness; Prognosis; Meta-analysis.

Contributions of each author:

Author 1 - Yi Zhang.

Author 2 - Hao Han.

Author 3 - Yunling Tian.

Author 4 - Jing Dong.

Author 5 - Yage Yu. 